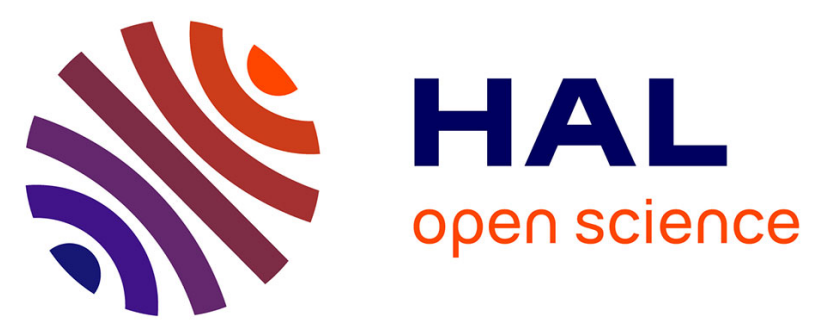

\title{
Propagation d'une onde électromagnétique dans un guide cylindrique rempli d'une substance anisotrope: application à la détermination des constantes diélectriques du quartz à $9000 \mathrm{MHz}$
}

\author{
D. Pradoux, F. Blanc, R. Fanguin, G. Raoult
}

\section{To cite this version:}

D. Pradoux, F. Blanc, R. Fanguin, G. Raoult. Propagation d'une onde électromagnétique dans un guide cylindrique rempli d'une substance anisotrope: application à la détermination des constantes diélectriques du quartz à $9000 \mathrm{MHz}$. Revue de Physique Appliquée, 1971, 6 (2), pp.223-227. 10.1051/rphysap:0197100602022300 . jpa-00243531

\section{HAL Id: jpa-00243531 https://hal.science/jpa-00243531}

Submitted on 1 Jan 1971

HAL is a multi-disciplinary open access archive for the deposit and dissemination of scientific research documents, whether they are published or not. The documents may come from teaching and research institutions in France or abroad, or from public or private research centers.
L'archive ouverte pluridisciplinaire HAL, est destinée au dépôt et à la diffusion de documents scientifiques de niveau recherche, publiés ou non, émanant des établissements d'enseignement et de recherche français ou étrangers, des laboratoires publics ou privés. 


\title{
PROPAGATION D'UNE ONDE ÉLECTROMAGNÉTIQUE DANS UN GUIDE CYLINDRIQUE REMPLI D'UNE SUBSTANCE ANISOTROPE : APPLICATION A LA DÉTERMINATION DES CONSTANTES DIÉLECTRIQUES DU QUARTZ A 9000 MHz
}

\author{
par D. PRADOUX, F. BLANC, R. FANGUIN et G. RAOULT \\ Laboratoire de Radioélectricité, Faculté des Sciences, Clermont-Ferrand
}

\begin{abstract}
Résumé. - Il n'est pas possible de résoudre en coordonnées cylindriques les équations de Maxwell en milieu anisotrope. Nous avons réduit le calcul au cas de deux plans méridiens particuliers, le calcul littéral peut alors être fait jusqu'au bout. Nous avons calculé les écarts de longueur d'onde guidée. En tenant compte des réflexions multiples dans un cristal de quartz, et en utilisant la méthode précédente, nous avons établi un programme qui nous a permis de tracer des courbes théoriques que nous avons comparées aux courbes expérimentales. Les constantes diélectriques du quartz à $10 \mathrm{GHz}$ sont respectivement 4,51 et 4,63 .
\end{abstract}

Abstract. - It is impossible to solve in cylindrical coordinates the Maxwell equations in an anisotropic medium. We reduced the operation to the case of two particular meridian planes. We calculated the differences between guided wavelengths. Having taken into account the multiple reflexions in a quartz cristal, and using the preceeding method, we made a program which allowed us to draw theoretical curves to be compared to the experimental ones. The values of the dielectric constants of quartz at $10 \mathrm{GHz}$ are 4,51 and 4,63.

Introduction. - Notre laboratoire a étudié le cas de la propagation anisotrope dans un guide à section rectangulaire [1], [5]. Ce cas peut être traité complètement de façon littérale et nous avons fait de nombreuses vérifications expérimentales pour montrer le bien-fondé de nos calculs.

Dans le cas des guides à section circulaire, cette résolution littérale n'est plus possible. Nous avons étudié expérimentalement l'action sur une onde $\mathrm{TE}_{11}$ d'un cristal de quartz ou de spath parallèles en bande $X$ [2], [3], [4]. Une première interprétation élémentaire a été donnée de ces expériences [6] en appliquant les principes de l'optique des ondes TEM. Il subsiste cependant des difficultés, et c'est pourquoi nous avons essayé de pousser le plus loin possible la résolution littérale. Ayant introduit la constante diélectrique sous forme matricielle, car nous nous limitons aux effets linéaires, nous écrirons les équations de Maxwell en coordonnées cylindriques. Nous avons simplifié le problème en supposant que l'un des axes électriques de la substance est suivant la direction de propagation. Par élimination successive des composantes de $E$ et de $H$, nous pouvons nous ramener à deux équations entre $E_{z}$ et $H_{z}$ que nous donnons dans l'annexe. Ces équations ne peuvent être manipulées car des dérivées croisées ne s'éliminent pas et nous nous trouvons bloqués.

Il est en revanche possible de chercher à résoudre ces équations dans deux plans méridiens passant par les deux autres axes électriques. Les équations se simplifient, et peuvent ainsi nous donner les valeurs des composantes des champs dans ces plans. Nous ne connaissons pas la forme des champs en dehors de ces plans qui doivent être des combinaisons plus ou moins compliquées des solutions trouvées, mais notre méthode nous permet de connaître toutes les solutions dans ces plans.

Etude des différents modes. - Dans le cas des substances isotropes, la théorie de G. Goubau [7] montre que le passage du guide vide au milieu considéré se fait avec conservation de mode. Il est vraisemblable de penser que dans le cas d'une substance anisotrope, le mode se conserve aussi, tout au moins au voisinage des plans méridiens, contenant les axes électriques qui sont des plans de symétrie. Nous résoudrons donc en modes TE et TM et par continuité, nous supposerons qu'un mode TE donne naissance à un mode TE; de même pour TM.

La constante diélectrique s'écrira pour un axe faisant l'angle avec Ox.

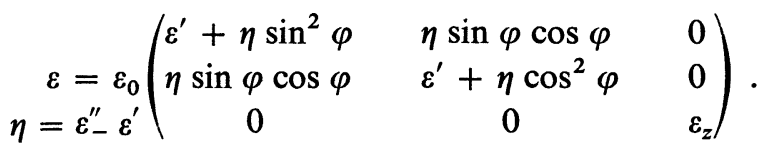

CAS DES mOdes TE. - Nous ferons alors $E_{z}=0$ et nous aurons deux équations aux dérivées partielles en $H_{z}$. Par manipulations, dans chaque cas : $\varphi=0$ ou $\varphi=\pi / 2$ nous pourrons ramener cette équation à une équation de Bessel d'ordre $v$ voisin d'un nombre entier $n$. Le champ sera donc décrit par des fonctions extrême- 
ment voisines de celles obtenues pour un guide renfermant une substance isotrope. Les conditions aux limites donneront les constantes de propagation. Précisons : si nous considérons la solution pour $\varphi=\pi / 2$ le champ $E$ se trouve dans le plan $\varphi=0$ et pour l'axe Ox, la constante diélectrique est $\varepsilon^{\prime}$. Nous aurons comme solution une fonction de Bessel extrêmement voisine de celle que nous obtiendrions dans un guide rempli d'une substance isotrope de constante diélectrique $\varepsilon^{\prime}$. Si maintenant nous considérons la solution pour $\varphi=0$, le champ $E$ se trouve dans un plan $\varphi=\pi / 2$ et suivant l'axe $O y$, la constante diélectrique est $\varepsilon^{\prime \prime}$. Nous aurons comme solution une fonction de Bessel extrêmement voisine de celle que nous obtiendrions pour un guide rempli d'une substance isotrope de constante diélectrique $\varepsilon^{\prime \prime}$. Nous avons calculé la variation relative de longueur d'onde guidée que nous devons observer dans les deux cas. Elles sont en première approximation égales et opposées.

$$
\frac{\mathrm{d} \lambda_{g}}{\lambda_{g}}=\left(\frac{\lambda_{g}}{\lambda_{0}}\right)^{2} \frac{1}{n_{0}^{2}-Z_{0}^{2}}\left[n Z_{0} \frac{\frac{\mathrm{d} J_{n}^{\prime}\left(Z_{0}\right)}{\mathrm{d} n}}{J_{n}\left(Z_{0}\right)}-1\right]\left(\varepsilon^{\prime \prime}-\varepsilon^{\prime}\right) .
$$

Dans le cas d'un mode $\mathrm{TE}_{11}$ : pour $n=1$ et la première racine $Z_{0}=1,8412 \mathrm{de} J^{\prime}(Z)=0$, avec $\varepsilon^{\prime \prime}-\varepsilon^{\prime}=0,05 ; \mathrm{d} \lambda_{g} / \lambda_{g}=0,008$, ce qui est très faible.

CAS DES MODES TM. - Nous faisons maintenant $H_{z}=0$ et nous opérons de la même manière : nous arrivons ainsi à une équation de Bessel d'ordre $v^{\prime}$ très voisin d'un entier $n$. Le champ sera donc décrit par une fonction de Bessel extrêmement voisine de celles obtenues pour un guide rempli de substance isotrope. Les conditions aux limites permettent de calculer les constantes de propagation.

De même que pour les modes TE, il y aura deux types de propagation et l'écart des longueurs d'onde guidées ne sera plus cette fois le même en valeur absolue. Nous aurons :

- pour le champ $E$ dans le plan $\varphi=0$

$$
\frac{\mathrm{d} \lambda_{g}}{\lambda_{g}} \#-\left[\frac{A_{0}^{\prime}}{2 k_{0}^{2}}+\frac{n}{2 A_{0}^{\prime} R} \frac{\frac{\mathrm{d} J_{n}\left(Z_{0}\right)}{\mathrm{d} n}}{J_{n}^{\prime}\left(Z_{0}\right)}\right] \frac{\varepsilon^{\prime \prime}-\varepsilon^{\prime}}{\varepsilon^{\prime}}
$$

- pour le champ $E$ dans le plan $\varphi=\pi / 2$

$$
\frac{\mathrm{d} \lambda_{g}}{\lambda_{g}} \#-\left[\frac{n}{2 A_{0}^{\prime \prime} R} \frac{\frac{\mathrm{d} J_{n}\left(Z_{0}\right)}{\mathrm{d} n}}{J_{n}^{\prime}\left(Z_{0}\right)}\right] \frac{\varepsilon^{\prime \prime}-\varepsilon^{\prime}}{\varepsilon^{\prime \prime}}
$$

les variations sont encore très faibles : pour $n=1$ et la première racine ; on trouve 0,007 dans le premier cas et 0,003 dans le second cas.

Application à la mesure des constantes diélectriques du quartz. - Nous plaçons dans un guide à section circulaire un morceau de quartz parallèle, l'axe étant pris comme axe Ox, la longueur de ce quartz étant $d$. $\varepsilon^{\prime}$ sera la constante diélectrique pour $E$ suivant l'axe, c'est la constante extraordinaire. $\varepsilon^{\prime \prime}$ sera pour le champ $E$ perpendiculaire à l'axe, c'est la constante ordinaire.

Nous aurons pour chacune de ces ondes des coefficients de réflexion aux extrémités du cristal $\rho^{\prime}$ et $\rho^{\prime \prime}$; des déphasages dans les propagations $\varphi^{\prime}$ et $\varphi^{\prime \prime}$. Un calcul classique nous permet de connaître l'amplitude émergente par superposition des ondes réfléchies multiples.

$$
x=A \frac{\left(1-\rho^{2}\right) \mathrm{e}^{-j \varphi}-\rho}{1-\rho^{2} \mathrm{e}^{-2 j \varphi}} .
$$

Les coefficients de réflexion sont calculables par la formule

$$
\rho=\frac{u-v}{u+v}
$$

avec

$$
u=\sqrt{\varepsilon(\varepsilon-B)}, v=\sqrt{1-B} \quad \text { et } \quad B=\frac{\lambda^{2}}{\lambda_{\mathrm{c}}^{2}}
$$

$\lambda_{\mathrm{c}}$ étant la longueur d'onde de coupure du guide vide.

En posant

$$
\begin{aligned}
& \varepsilon^{\prime}=\varepsilon-\mathrm{d} \varepsilon \\
& \varepsilon^{\prime \prime}=\varepsilon+\mathrm{d} \varepsilon .
\end{aligned}
$$

Il est facile de calculer $\rho+\mathrm{d} \rho$ et $\rho-\mathrm{d} \rho ; \varphi-\mathrm{d} \varphi$, $\varphi+\mathrm{d} \varphi$ grâce à des développements limités. Nous avons besoin de l'angle $\delta$ de déphasage entre les deux composantes à la sortie du quartz

$$
\begin{aligned}
& \left(1-\rho^{\prime 2}\right)\left(1+\rho^{\prime \prime 2}\right) \cos \varphi^{\prime} \cos \varphi^{\prime \prime}-\left(1+\rho^{\prime 2}\right) \times \\
& \operatorname{tg} \delta=\frac{\times\left(1+\rho^{\prime \prime 2}\right) \sin \varphi^{\prime} \sin \varphi^{\prime \prime}}{\left(1-\rho^{\prime 2}\right)\left(1-\rho^{\prime \prime 2}\right) \cos \varphi^{\prime} \cos \varphi^{\prime \prime}+\left(1+\rho^{\prime 2}\right) \times} \\
& \times\left(1+\rho^{\prime \prime 2}\right) \sin \varphi^{\prime} \sin \varphi^{\prime \prime}
\end{aligned}
$$

l'angle $\alpha$ que fait l'axe de la vibration elliptique avec Ox.

$$
\operatorname{tg} 2 \alpha=\operatorname{tg} 2 \gamma \cos \delta \quad \operatorname{tg} \gamma=B_{1} / B_{2}
$$

avec

$$
\begin{aligned}
& B_{1}=-\frac{E \cos i\left(1-\rho^{\prime 2}\right)}{\sqrt{1+\rho^{\prime 4}-2 \rho^{\prime 2} \cos 2 \varphi^{\prime}}} \\
& B_{2}=\frac{E \sin i\left(1-\rho^{\prime \prime 2}\right)}{\sqrt{1+\rho^{\prime \prime}-2 \rho^{\prime \prime} \cos 2 \varphi^{\prime \prime}}} .
\end{aligned}
$$

Etude du cas de rotation nulle. - Considérons le cas où $\alpha=i$. Si, en première approximation et à juste titre, nous faisons $\cos \delta=1$, nous en tirons $\gamma=i$ et comme $\operatorname{tg} \gamma=\operatorname{tg} i\left(1+m \frac{\mathrm{d} \varepsilon}{\varepsilon}\right)$ cela nous donne $m=0$. Le calcul de $m$ est fastidieux, mais sans difficultés. C'est une fraction dont le dénominateur reste fini. Le numérateur s'écrit :

$$
\sin 2 \varphi-A \cos 2 \varphi-A=N \text {. }
$$

Avec

$$
A=\frac{u^{2}+v^{2}}{u^{2}-v^{2}} \times \frac{u^{2}+\varepsilon^{2}}{\varepsilon^{2}} \times \frac{1}{\rho} .
$$


Pour résoudre l'équation $N=0$, le plus simple est de prendre l'intersection du cercle de rayon un avec la droite $y-A(x-1)=0$.

Une solution est immédiate :

$$
x=1 ; y=0 ; 2 \varphi=2 k \pi .
$$

L'autre se calcule de manière approchée car $A$ est petit. On trouve

$$
2 \varphi \# \pi+\frac{\lambda}{\pi d}+2 k \pi .
$$

Détermination numérique des constantes diélectriques. - Il faut faire un choix de la valeur moyenne de $\varepsilon$ et le cas de rotation nulle nous servira. Nous avons considéré les courbes de rotation nulle quel que soit $i$. Pour deux cristaux différents :

$$
\begin{array}{rlrl}
d & =40 \mathrm{~mm} & d & =30 \mathrm{~mm} \\
R & =11,40 \mathrm{~mm} & R & =11,40 \mathrm{~mm} \\
k & =9 & k & =7 \\
f & =8720 \mathrm{MHz} & f & =9050 \mathrm{MHz} \\
\varepsilon & =4,58 & \varepsilon & =4,57 .
\end{array}
$$

On remarquera la concordance exceptionnelle, due au terme correctif de la deuxième solution. Nous choisirons comme valeur moyenne 4,57. Les calculs ont été faits par l'ordinateur du centre de calcul numérique de la Faculté des Sciences de Clermont-Ferrand. Nous avons considéré deux séquences de valeurs; pour $\varepsilon$, de 0,10 en 0,10 et pour d $\varepsilon ; 0,03 ; 0,07 ; 0,11 ; 0,15 ; 0,17$. L'ordinateur a calculé les valeurs des rotations $\theta$ et ellipticités $\operatorname{tg} \beta$ pour différentes longueurs d'onde et différents angles $i$. Nous avons ensuite tracé point par point les courbes de variation en fonction de $i$ pour chaque $\lambda$. Ces courbes théoriques ont été ensuite comparées aux courbes expérimentales; l'écart minimum entre elles est obtenu pour $\varepsilon=4,57$ et $\mathrm{d} \varepsilon=0,06$, ce chiffre étant obtenu par interpolation.

Nous en déduisons :

$$
\begin{aligned}
& \varepsilon^{\prime}=4,51 \pm 0,05 \\
& \varepsilon^{\prime \prime}=4,63 \pm 0,05 .
\end{aligned}
$$

Ces erreurs que nous indiquons sont calculées et sont certainement largement supérieures aux erreurs effectives qui doivent vraisemblablement être voisines de 0,03 .
Conclusion. - L'étude théorique a justifié une manière simple de procéder qui consiste à considérer deux vibrations rectangulaires comme se propageant dans deux milieux isotropes différents. Un calcul nous a permis alors de déterminer les angles des axes, les ellipticités des vibrations émergent d'un cristal de quartz. Une coïncidence avec les réseaux de courbes obtenues expérimentalement nous a permis de calculer les constantes diélectriques du quartz (à environ un pour cent près). Ces valeurs 4,51 et 4,63 sont très voisines des valeurs statiques connues $(8,9)$ et semblent prouver que la permittivité du quartz varie peu avec la fréquence jusqu'aux longueurs d'ondes centimétriques.

Annexe. - Les équations de départ sont, en coordonnées cylindriques, pour des champs à la pulsation $\omega$

$$
\begin{aligned}
& \frac{1}{\rho} \frac{\partial E_{z}}{\partial \varphi}-\frac{\partial E_{\varphi}}{\partial Z}=-j \mu \mu_{0} \omega H_{\rho} \\
& \frac{\partial E_{\rho}}{\partial Z}-\frac{\partial E_{z}}{\partial \rho}=-j \mu \mu_{0} \omega H_{\varphi} \\
& \frac{1}{\rho} \frac{\partial\left(\rho E_{\varphi}\right)}{\partial \rho}-\frac{\partial E_{\rho}}{\rho \partial \varphi}=-j \mu \mu_{0} \omega H_{z}
\end{aligned}
$$

$\frac{1}{\rho} \frac{\partial H_{z}}{\partial \varphi}-\frac{\partial H_{\varphi}}{\partial Z}=$

$$
=j \omega \varepsilon_{0}\left[\left(\varepsilon^{\prime}+\eta \sin ^{2} \varphi\right) E_{\rho}+\eta \sin \varphi \cos \varphi E_{\varphi}\right]
$$

$$
\begin{aligned}
& \frac{\partial H_{\rho}}{\partial Z}-\frac{\partial H_{z}}{\partial \rho}= \\
& =j \omega \varepsilon_{0}\left[\eta \sin \varphi \cos \varphi E_{\rho}+\left(\varepsilon^{\prime}+\eta \cos ^{2} \varphi\right) E_{\varphi}\right] \\
& \frac{1}{\rho} \frac{\partial\left(\rho H_{\varphi}\right)}{\partial \rho}-\frac{\partial H_{\rho}}{\rho \partial \varphi}=j \omega \varepsilon_{0} \varepsilon_{z} E_{z}
\end{aligned}
$$

En tirant $E_{\rho}$ et $E_{\varphi}$ de 4 et 5, en portant dans (1) et (2), il est facile d'éliminer les quantités intermédiaires avec (6) et d'avoir une première relation entre $H_{z}$ et $E_{z}$.

En opérant de même à partir de $H_{\rho}$ et $H_{\varphi}$ dans (1) et (2) et portant dans (4) et (5) par diminution avec (3) on a une deuxième relation entre $H_{z}$ et $E_{z}$. Ces relations s'écrivent :

$$
\begin{aligned}
& J\left\{\left[\frac{\varepsilon_{0} \varepsilon^{\prime} \varepsilon_{z}}{\varepsilon^{\prime \prime}}\right.\right.\left(\omega^{4} \varepsilon^{\prime 2} \mu^{2} \mu_{0}^{2} \varepsilon_{0}^{2}+\varepsilon_{0} \frac{\varepsilon^{\prime}+\varepsilon^{\prime \prime}}{\varepsilon^{\prime}} \omega^{2} \varepsilon^{\prime \prime} \mu \mu_{0} \frac{\partial^{2}}{\partial Z^{2}}+\frac{\varepsilon^{\prime \prime}}{\varepsilon^{\prime}} \frac{\partial^{4}}{\partial Z^{4}}\right)+\varepsilon_{0} \eta \frac{\partial^{2}}{\partial Z^{2}}\left\{\sin ^{2} \varphi \frac{1}{\rho} \frac{\partial}{\partial \rho}\left(\rho \frac{\partial}{\partial \rho}\right)+\right. \\
&\left.\left.+\frac{1}{\rho^{2}} \cos ^{2} \varphi \frac{\partial^{2}}{\partial \varphi^{2}}+\frac{1}{\rho}\left[\frac{\partial}{\partial \rho}\left(\sin 2 \varphi \frac{\partial}{\partial \varphi}+\cos 2 \varphi\right)-\frac{1}{\rho} \sin 2 \varphi \frac{\partial}{\partial \varphi}\right]\right\}\right]+\varepsilon_{0} \varepsilon^{\prime}\left(\frac{\partial^{2}}{\partial Z^{2}}+\omega^{2} \varepsilon_{0} \mu_{0} \varepsilon^{\prime \prime} \mu\right) \\
&\left.\times\left[\frac{1}{\rho} \frac{\partial}{\partial \rho}\left(\rho \frac{\partial}{\partial \rho}\right)+\frac{1}{\rho^{2}} \frac{\partial^{2}}{\partial \varphi^{2}}\right]\right\} E_{z}-\omega^{2} \mu \mu_{0} \varepsilon_{0} \eta \frac{1}{\rho} \frac{\partial}{\partial Z}\left[\frac{1}{2} \sin 2 \varphi \frac{\partial}{\partial \rho}\left(\rho \frac{\partial}{\partial \rho}-\frac{1}{\rho} \frac{\partial^{2}}{\partial \varphi^{2}}\right)+\right. \\
&\left.+\cos 2 \varphi\left(\frac{\partial}{\partial \rho}-\frac{1}{\rho}\right)-\sin 2 \varphi \frac{\partial}{\partial \varphi}\right] H_{z}
\end{aligned}
$$


et

$$
\begin{gathered}
j\left\{\left(\frac{\partial^{2}}{\partial Z^{2}}+\omega^{2} \mu \mu_{0} \varepsilon_{0} \varepsilon^{\prime}\right)\left(\frac{1}{\rho} \frac{\partial}{\partial \rho}\left[\rho \frac{\partial}{\partial \rho}\right]+\frac{1}{\rho^{2}} \frac{\partial^{2}}{\partial \varphi^{2}}+\frac{\partial^{2}}{\partial Z^{2}}+\omega^{2} \mu \mu_{0} \varepsilon^{\prime} \varepsilon_{0}\right)+\omega^{2} \mu \mu_{0} \varepsilon_{0} \eta\left[\sin ^{2} \varphi \frac{1}{\rho} \frac{\partial}{\partial \rho}\left(\rho \frac{\partial}{\partial \rho}\right)+\right.\right. \\
\left.\left.+\cos ^{2} \varphi \frac{1}{\rho^{2}} \frac{\partial^{2}}{\partial \varphi^{2}}+\frac{\partial^{2}}{\partial Z^{2}}+\omega^{2} \mu \mu_{0} \varepsilon^{\prime} \varepsilon_{0}+\frac{1}{\rho}\left(\frac{\partial}{\partial \rho}\left[\sin 2 \varphi \frac{\partial}{\partial \varphi}+\cos 2 \varphi\right]-\frac{1}{\rho} \sin 2 \varphi \frac{\partial}{\partial \varphi}\right)\right]\right\} H_{z} \\
-\omega^{2} \varepsilon_{0} \eta \frac{1}{\rho} \frac{\partial}{\partial Z}\left\{\frac{1}{2} \sin 2 \varphi\left[\frac{\partial}{\partial \rho}\left(\rho \frac{\partial}{\partial \rho}\right)-\frac{1}{\rho} \frac{\partial^{2}}{\partial \varphi^{2}}\right]+\cos 2 \varphi \frac{\partial}{\partial \varphi}\left(\frac{\partial}{\partial \rho}-\frac{1}{\rho}\right)-\sin 2 \varphi \frac{\partial}{\partial \rho}\right\} E_{z}=0
\end{gathered}
$$

Mode TE; $\varphi=0$. Nous faisons $E_{z}=0$ et $\varphi=0$ dans les équations précédentes il vient :

$$
\begin{gathered}
\frac{\partial^{2}}{\partial Z \partial \varphi}\left(\frac{\partial}{\partial \rho}-\frac{1}{\rho}\right) H_{z}=0 \\
\left\{\left(\frac{\partial^{2}}{\partial Z^{2}}+\omega^{2} \mu \mu_{0} \varepsilon^{\prime} \varepsilon_{0}\right)\left[\frac{1}{\rho} \frac{\partial}{\partial \rho}\left(\rho \frac{\partial}{\partial \rho}\right)+\frac{1}{\rho^{2}} \frac{\partial^{2}}{\partial \varphi^{2}}+\frac{\partial^{2}}{\partial Z^{2}}+\omega^{2} \mu \mu_{0} \varepsilon^{\prime} \varepsilon_{0}\right]+\right. \\
+\omega^{2} \mu \mu_{0} \varepsilon_{0} \eta\left(\frac{1}{\rho^{2}} \frac{\partial^{2}}{\partial \varphi^{2}}+\frac{\partial^{2}}{\partial Z^{2}}+\omega^{2} \mu \mu_{0} \varepsilon^{\prime} \varepsilon_{0}+\frac{1}{\rho} \frac{\partial}{\partial \rho}\right) H_{z}=0
\end{gathered}
$$

En posant :

$$
H_{z}=H_{0} R(\rho) \Phi(\varphi) \mathrm{e}^{j(\omega t-k Z)}
$$

et

$$
A^{\prime 2}=\omega^{2} \mu \mu_{0} \varepsilon^{\prime} \varepsilon_{0}-k^{2} ; A^{\prime \prime 2}=\omega^{2} \mu \mu_{0} \varepsilon^{\prime \prime} \varepsilon_{0}-k^{2}
$$

il vient :

$$
\left[\frac{1}{\rho} \frac{\partial}{\partial \rho}+\frac{A^{\prime 2}}{A^{\prime \prime 2}} \frac{\partial^{2}}{\partial \rho^{2}}+\frac{1}{\rho^{2}} \frac{\partial^{2}}{\partial \varphi^{2}}+A^{\prime 2}\right] H_{z}=0 .
$$

Nous en concluons que $\Phi=\mathrm{e}^{ \pm j n \varphi} ; n$ entier et en posant $R(\rho)=\rho^{\alpha} u(\rho)$ avec

$$
2 \alpha=1-\frac{A^{\prime 2}}{A^{\prime 2}} \quad \text { et } \quad A^{\prime \prime} \rho=r,
$$

l'équation s'écrit :

$$
\frac{\mathrm{d}^{2} u}{\mathrm{~d} r^{2}}+\frac{1}{r} \frac{\mathrm{d} u}{\mathrm{~d} r}+\left[1+\frac{\alpha^{2}+n^{2}(1-2 \alpha)}{r^{2}}\right] u=0 .
$$

C'est une équation de Bessel d'ordre $v$ tel que $v^{2}=\alpha^{2}+n^{2}(1-2 \alpha)$.

La solution est alors $H_{z}=H_{0} \rho^{\alpha} J_{v}\left(A^{\prime \prime} \rho\right)$.

Il faut éliminer les fonctions $Y$ qui sont infinies sur l'axe et la dérivée par rapport à $\varphi$ de $H_{z}$ étant nulle pour $\varphi=0, H_{z}$ doit être un cosinus qui pour $\varphi=0$ vaut l'unité.

Conditions aux limites. - Il suffit d'écrire $\partial E_{z} / \partial \rho=0$ pour $\rho=R$, soit

$$
A^{\prime \prime} R J_{v}^{\prime}\left(A^{\prime \prime} R\right)+\alpha J_{v}\left(A^{\prime \prime} R\right)=0
$$

comme $v$ est voisin de $n$, considérons le mode TE de même $n$ et posons

$A_{0}^{\prime 2}=\omega^{2} \mu \mu_{0} \varepsilon^{\prime} \varepsilon_{0}-k_{0}^{2} ; \quad A_{0}^{\prime \prime 2}=\omega^{2} \mu \mu_{0} \varepsilon^{\prime \prime} \varepsilon_{0}-k_{0}^{2}$ avec

$$
A_{0}^{\prime \prime} R=Z_{0} \quad \text { et } \quad A^{\prime \prime} R=Z .
$$

Nous comparons l'équation

$$
Z J_{v}^{\prime}(Z)-\alpha J_{v}(Z)=0
$$

avec

$$
J_{n}^{\prime}\left(Z_{0}\right)=0
$$

Les racines $Z$ de l'équation seront voisines de $Z_{0}$ en posant $Z=Z_{0}+\mathrm{d} Z$ et en faisant des développements nous calculerons $\mathrm{d} Z$

$$
\mathrm{d} Z=\frac{\alpha Z_{0}}{n^{2}-Z_{0}^{2}}\left[1-n Z_{0} \frac{\frac{\mathrm{d} J_{n}^{\prime}\left(Z_{0}\right)}{\mathrm{d} n}}{J_{n}\left(Z_{0}\right)}\right]
$$

comme

$$
J^{\prime \prime}\left(Z_{0}\right)=\frac{n^{2}-Z_{0}^{2}}{Z_{0}^{2}} J_{n}\left(Z_{0}\right)
$$

de l'expression de

$$
\alpha \# \frac{\omega^{2} \mu \mu_{0}\left(\varepsilon^{\prime}-\varepsilon^{\prime \prime}\right) \varepsilon_{0}}{A_{0}^{\prime 2}}
$$

et de $\mathrm{d} Z$ nous déduisons

$$
\frac{\mathrm{d} k}{k_{0}}=-\frac{\mathrm{d} \lambda_{g}}{\lambda_{g}}=\frac{Z_{0} \mathrm{~d} Z}{K_{0}^{2} R^{2}}
$$

et

$$
\frac{\partial \lambda_{g}}{\lambda_{g}}=\left(\frac{\lambda_{g}}{\lambda_{0}}\right)^{2} \frac{1}{n^{2}-Z_{0}^{2}}\left[n Z_{0} \frac{\frac{\mathrm{d} J_{n}^{\prime}\left(Z_{0}\right)}{\mathrm{d} n}}{J_{n}\left(Z_{0}\right)}-1\right]\left(\varepsilon^{\prime \prime}-\varepsilon^{\prime}\right) .
$$

Le même type de raisonnement conduit aux autres formules. 


\section{Bibliographie}

[1] Bouche (A.), Diplôme d'Etudes Supérieures, ClermontFerrand, 1965.

[2] Bergerat (A.), Diplôme d'Etudes Supérieures, Clermont-Ferrand, 1966.

[3] Galateau (D.), Diplôme d'Etudes Supérieures, Clermont-Ferrand, 1965.

[4] Bessege (R.), Diplôme d'Etudes Supérieures, ClermontFerrand, 1965.
[5] Forest (A.), Diplôme d'Etudes Supérieures, ClermontFerrand, 1966.

[6] Blanc (F.), Fanguin (R.), Raoult (G.), Interélectronique, 1969, 4, 46 à 54 .

[7] Goubau (G.), Electromagnetic wave guides and cavities, Londres, 1961.

[8] Handbook of Chemistry and Physics, 46 ${ }^{\mathrm{e}}$ Edition, 196566.

[9] Errera, Polarisation diélectrique, P. U. F., 1928. 